\title{
SCALE-DEPENDENT VARIATION IN CORAL COMMUNITY SIMILARITY ACROSS SITES, ISLANDS, AND ISLAND GROUPS
}

\author{
Howard V. Cornell, ${ }^{1,4}$ Ronald H. Karlson, ${ }^{2}$ and Terence P. Hughes ${ }^{3}$ \\ ${ }^{1}$ Department of Environmental Science and Policy, University of California, One Shields Avenue, Davis, California 95616 USA \\ ${ }^{2}$ Department of Biological Science, University of Delaware, Newark, Delaware 19716 \\ ${ }^{3}$ ARC Centre of Excellence for Coral Reef Studies, James Cook University, Townsville, Queensland 4811 Australia
}

\begin{abstract}
Community similarity is the proportion of species richness in a region that is shared on average among communities within that region. The slope of local richness $(\alpha$ diversity) regressed on regional richness ( $\gamma$ diversity) can serve as an index of community similarity across regions with different regional richness. We examined community similarity in corals at three spatial scales (among transects at a site, sites on an island, and islands within an island group) across a $10000-\mathrm{km}$ longitudinal diversity gradient in the west-central Pacific Ocean. When $\alpha$ diversity was regressed on $\gamma$ diversity, the slopes, and thus community similarity, increased with scale $(0.085,0.261$, and 0.407 , respectively) because a greater proportion of $\gamma$ diversity was subsumed within $\alpha$ diversity as scale increased. Using standard randomization methods, we also examined how community similarity differed between observed and randomized assemblages and how this difference was affected by spatial separation of species within habitat types and specialization of species to three habitat types (reef flats, crests, and slopes). If spatial separation within habitat types and/or habitat specialization (i.e., underdispersion) occurs, fewer species are shared among assemblages than the random expectation. When the locations of individual coral colonies were randomized within and among habitat types, community similarity was $46-47 \%$ higher than that for observed assemblages at all three scales. We predicted that spatial separation of coral species within habitat types should increase with scale due to dispersal/extinction dynamics in this insular system, but that specialization of species to different habitat types should not change because habitat differences do not change with scale. However, neither habitat specialization nor spatial separation within habitat types differed among scales. At the two larger scales, each accounted for $22-24 \%$ of the difference in community similarity between observed and randomized assemblages. At the smallest scale (transect-site), neither spatial separation within habitat types nor habitat specialization had significant effects on community similarity, probably due to the small size of transect samples. The results suggest that coral species can disperse among islands in an island group as easily as they can among sites on an island over time scales that are relevant to their establishment and persistence on reefs.
\end{abstract}

Key words: $\alpha$ diversity; $\beta$ diversity; community similarity; coral; dispersal limitation; $\gamma$ diversity; underdispersion.

\section{INTRODUCTION}

The kinds of species that occur in ecological communities often differ from community to community within a defined geographical region. The total number of species within the region can thus be thought of as comprising two components: the mean number of species in a community and the degree of dissimilarity among communities (species turnover). These components have been designated $\alpha$ and $\beta$ diversity, respectively, and the species richness of the entire region has been designated $\gamma$ diversity (Whittaker 1960). All three components of diversity are based exclusively on species presence or absence. An additive relationship among these components,

Manuscript received 5 May 2006; revised 7 December 2006; accepted 5 January 2007. Corresponding Editor: M. A. Hixon.

${ }^{4}$ E-mail: hvcornell@ucdavis.edu

$$
\beta=\gamma-\alpha
$$

has recently been proposed by Lande (1996) and has become widely used in the literature (e.g., Loreau 2000, Wagner et al. 2000, Gering and Crist 2002, Gering et al. 2003, Crist et al. 2004, Okuda et al. 2004, Freestone and Inouye 2006).

This relationship may vary with $\gamma$ diversity (e.g., Koleff et al. 2003, Hillebrand 2004, Witman et al. 2004) and with spatial scale (Loreau 2000, Scheiner et al. 2000). A convenient method for studying this variation is to plot $\alpha$ vs. $\gamma$ diversity across a range of regions at each scale (Cornell and Lawton 1992, Loreau 2000, Gering and Crist 2002). In the present study, both local and regional scales vary. Thus, we define $\alpha$ diversity as the mean species richness within a set of samples pooled over a scale that has been chosen to define the community, the scale being indicated by a subscript. We define $\gamma$ diversity as the species richness in a larger 
set of samples pooled over a scale that has been chosen to define the region, the scale again being indicated by a subscript. The community scale is nested within the regional scale and for both $\alpha$ and $\gamma$ diversity, the larger the scale, the larger the set of pooled samples (see Methods). The slope ( $p$ ) of the plot of $\alpha$ vs. $\gamma$ diversity represents the mean proportion of species shared among communities across all regions. The higher the slope, the greater is the degree of species sharing. Thus, $p$ can be used as an index of mean community similarity across regions and as a basis for inferring how $\beta$ diversity varies with spatial scale.

If desired, a value similar to $\beta$ diversity but averaged over all values of $\gamma$ diversity can be recovered from $p$ using the expression

$$
\bar{\beta}_{1}=1-p .
$$

In this paper, we will report values for community similarity as well as $\bar{\beta}_{1}$.

If individuals of each species are scattered randomly within each region, then the slope of $\alpha$ vs. $\gamma$ diversity represents null (randomized) community similarity. Randomized community similarity can be higher than observed if species populations are on average underdispersed (clumped) or lower than observed if they are overdispersed (evenly spaced). Such deviations demand ecological explanations (Gotelli and Graves 1996). However, underdispersion is the rule in the coral assemblages considered here (Karlson et al. 2007), so overdispersion, which is extremely uncommon, will not be considered further.

Underdispersion can occur either because species are specialized to different habitat types (e.g., coral reef crest, coral reef slope, and so on) or because spatial separation of species occurs among locations within particular habitat types (Freestone and Inouye 2006). Henceforth, habitat type will be referred to as habitat for simplicity. Habitat specialization occurs either because species favor or survive better in some habitats vs. others, i.e., habitat specificity, or because species are so rare that there are insufficient individuals to occupy all habitats. Within-habitat spatial separation can result from range limits set by dispersal limitation, patchy extinction, aggregated settlement of propagules, facilitative or preemptive interactions among species, and other clustering processes. These processes cause species to occur in some locations but not others within a given type of habitat. Within-habitat spatial separation and habitat specialization have both been shown to be important in terrestrial and marine taxa (Harrison et al. 1992, Cody 1993, Harrison 1997, 1999, Ellingsen and Gray 2002, He and Legendre 2002, Green and Ostling 2003, Crist et al. 2004, Qian et al. 2004).

Underdispersion and the relative contributions of habitat specialization and within-habitat spatial separation to underdispersion may change with spatial scale. The degree of change may, in turn, depend upon the diversity and mean size of habitats, the mean dispersal ability of species in the pool, the scale of interspecific interactions, and so on. Several recent studies have explored the relationship between spatial scale and underdispersion, and consequently, community similarity among localities (Gering and Crist 2002, Rivadeneira et al. 2002, Summerville et al. 2003, Okuda et al. 2004, Freestone and Inouye 2006). Fewer have evaluated the relative contributions of habitat specialization and within-habitat spatial separation to community similarity at different scales (Crist et al. 2004, Freestone and Inouye 2006). None have evaluated these relative contributions using $\alpha$ vs. $\gamma$ diversity plots, although Gering and Crist (2002) used such plots to explore the relationship between spatial scale and community similarity.

In this study we used hierarchical randomization tests to explore the community similarity of coral assemblages at multiple spatial scales across a large portion of the west-central Pacific Ocean. We chose this system because $\beta$ diversity has been poorly studied in marine environments (Gaston and Williams 1996, Clarke and Lidgard 2000), and coral assemblages are no exception. Only two recent studies that we know of (Edinger et al. 2000, Connell et al. 2004) measured coral $\beta$ diversity, and neither study did measurements at multiple spatial scales. Moreover, corals conveniently exhibit a strong longitudinal diversity gradient in the Indo-Pacific (Hughes et al. 2002, Roberts et al. 2002, Connolly et al. 2003). Such a gradient is needed to examine changes in mean community similarity as $\gamma$ diversity changes.

Our hypotheses are as follows: (1) As the spatial scale at which $\alpha$ diversity is sampled increases, mean community similarity among samples should also increase. According to the species-area relationship, the larger the scale at which $\alpha$ diversity is sampled, the greater its value. Of course, $\gamma$ diversity also increases with scale. Nevertheless, a larger proportion of $\gamma$ diversity will be subsumed by $\alpha$ diversity because most species-area relationships on logarithmic scales have slopes that are less than 1 . This means that on arithmetic scales, the relationship is convex curvilinear. Therefore, diversity at larger scales (e.g., $\gamma$ diversity) should increase more slowly with increasing area than at smaller scales (e.g., a diversity). (2) Randomized community similarity should be higher than observed at all scales due to some combination of within-habitat spatial separation and habitat specialization. (3) As spatial scale increases, the effects of within-habitat spatial separation on observed community similarity should increase (scale dependent) because, as mean distances among locations increase at larger spatial scales, dispersal rate declines relative to the rate of local extinction, limiting species ranges. However, habitat specialization should be the same at each scale (scaleindependent) because differences among habitats do not change at larger scales. 


\section{Methods \\ Field sampling}

We sampled coral assemblages in the west-central Pacific Ocean from Indonesia eastward to the Society Islands (Karlson et al. 2004; see Plate 1). Coral species richness peaks in the so-called Indo-Australian hotspot and declines both longitudinally and latitudinally with distance from the hotspot (Connolly et al. 2003, Hughes et al. 2003). It ranges from $\sim 600$ species in Indonesia to $\sim 175$ species in the Society Islands.

Sampling was done over a period of four years (19992002) within five island groups (Indonesia, Papua New Guinea, Solomon Islands, American Samoa, and the Society Islands). We sampled three habitats (reef flat, crest, and slope) at three spatial scales in all island groups (Fig. 1). The three scales were transects within sites (transect-site), sites within islands (site-island), and islands within island groups (island-island group). In each island group, three different islands were selected. On each island, four sampling sites were chosen in each of the three habitat types at various locations around the fringing reef. Reef flats were sampled 5-10 m inshore of breaking waves, reef crests were sampled at depths of 1$2 \mathrm{~m}$, and reef slopes were sampled at depths of 6-7 $\mathrm{m}$ on the fore reef (see Plate 1). At each site, 10 10-m transect lines were draped over the substratum parallel to depth contours, and all coral colonies intercepted were identified to species level (Fig. 1).

\section{Tests of the hypotheses}

We determined species richness and community similarities at three spatial scales. We first calculated mean $\alpha$ diversity and $\gamma$ diversity at each scale (Fig. 1). The mean number of coral species per transect, per site (10 transects, pooled), and per island (40 transects, pooled) provided estimates of $\alpha$ diversity in each habitat at each progressively larger scale. To calculate $\gamma$ diversities, we first pooled transects over all three habitats. Thus, the total number of species per site (S; 30 transects, pooled), per island (I; 120 transects, pooled), and per island group (IG; 360 transects, pooled) provided estimates of $\gamma_{\mathrm{S}}, \gamma_{\mathrm{I}}$, and $\gamma_{\mathrm{IG}}$ diversity at each progressively larger scale (Fig. 1). We pooled habitats because our null expectation was that habitat type had no effect on species presence/absence, and thus the species occupying each habitat would be a random draw from the pool for all three. One of the hypotheses to be tested by the randomization models presented here is that habitat has an effect on species occurrences. Pooling of habitats allows us to quantify this effect. We then determined mean $\alpha$ diversities by averaging per habitat $\alpha$ diversity over all three habitats (Fig. 1). This was done to avoid pseudoreplication (more than one $\alpha$ diversity estimate within a single region; Srivastava 1998) and to standardize the number of $\alpha$ diversity values to that of the randomization among habitats (see Construction of random models). Finally, this mean $\alpha$

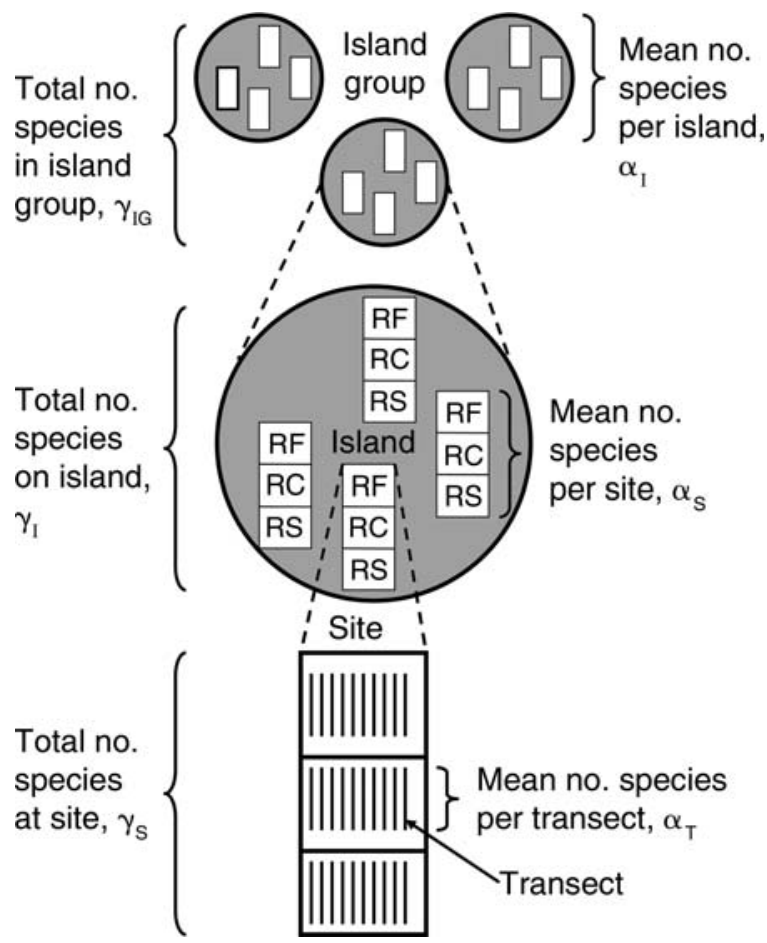

FIG. 1. Hierarchical sampling scheme for one of the five island groups sampled across the western Pacific. Gamma diversities at each scale were calculated by summing all of the species encountered at a site, on an island, or within an island group $\left(\gamma_{\mathrm{S}}, \gamma_{\mathrm{I}}, \gamma_{\mathrm{IG}}\right)$. Mean $\alpha$ diversities were calculated by determining $\alpha$ diversity for each habitat and then averaging across habitats at each scale: transect, site, and island $\left(\alpha_{\mathrm{T}}, \alpha_{\mathrm{S}}\right.$, $\alpha_{\mathrm{I}}$ ). Key to abbreviations: RF, reef flat; RC, reef crest; RS, reef slope.

diversity was regressed on $\gamma$ diversity at each scale and the slope of the regression was used as a measure of the observed community similarity $\left(p_{\mathrm{ob}}\right)$. If hypothesis 1 is correct (as the spatial scale at which $\alpha$ diversity is sampled increases, mean community similarity among samples should also increase), then $p_{\text {ob }}$ should progressively increase as spatial scale increases.

To test hypothesis 2 (randomized community similarity should exceed that observed at all scales), a random model removing the effects of underdispersion on $\alpha$ diversity at each scale was generated (see Construction of random models). Randomized $\alpha$ diversity was then plotted against $\gamma$ diversity to yield randomized community similarity, which was then compared to observed community similarity. Randomized community similarities that are higher than observed at all three scales would support hypothesis 2 because the combined effects of underdispersion at one scale are subsumed within observed $\alpha$ diversity at the next larger scale. Thus, the method detects additional underdispersion at higher scales above and beyond that at lower scales.

Hypothesis 3 (within-habitat spatial separation, but not habitat specialization, should increase with scale) was tested by generating one other randomization model 


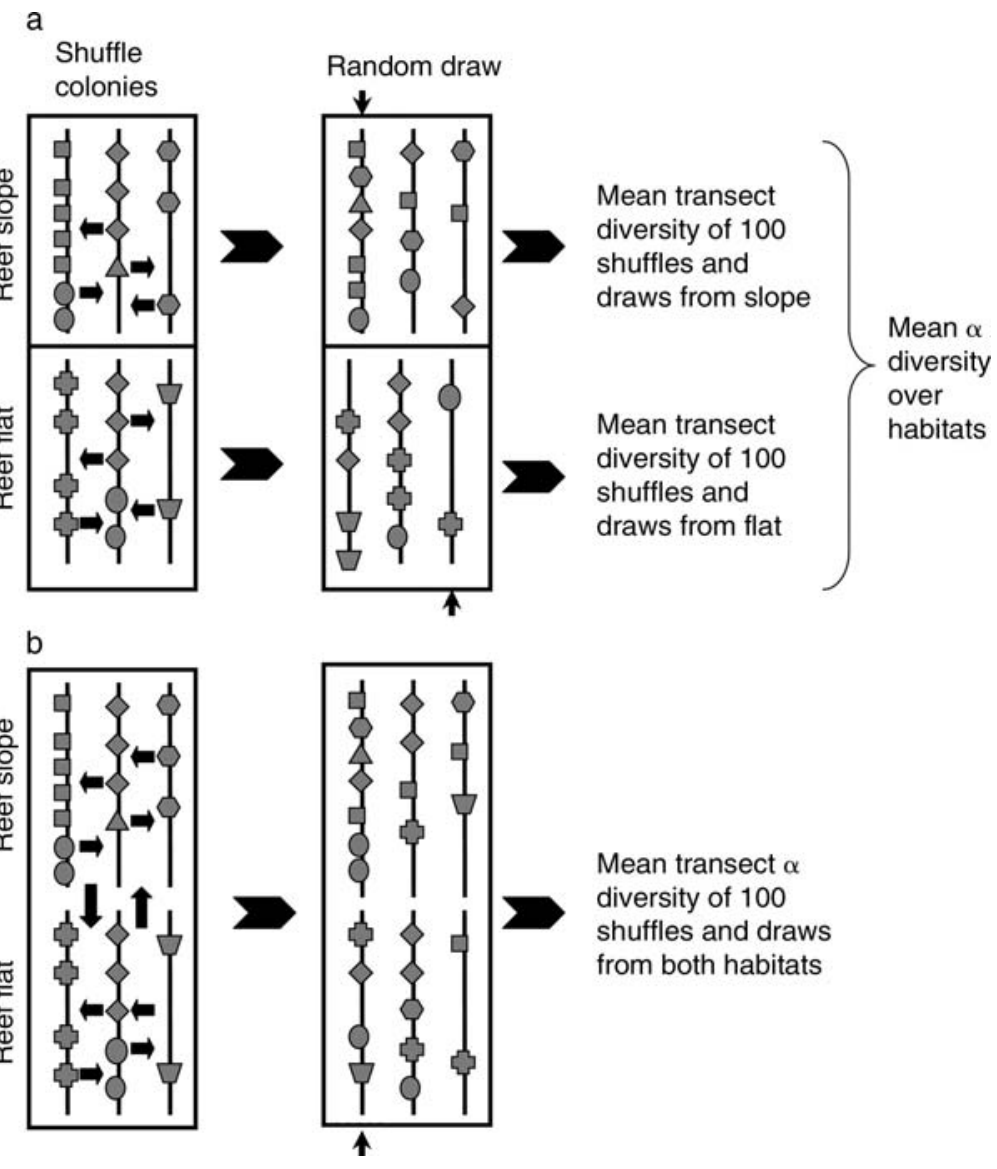

FIG. 2. Simplified randomization scheme (three transects instead of 10, two habitats instead of three) for removing the effects of underdispersion on community similarity at the transect-site spatial scale. Each of the three transect lines within a habitat intercepts a number of coral colonies. Different colony shapes represent different species. Small arrows indicate a random draw. For illustrative purposes, the coral species are maximally underdispersed (all colonies of a given species on one transect) at the start of the randomization. For details, see Methods: Construction of random models. (a) Colonies are shuffled among transects within each habitat to remove within-habitat spatial separation. (b) Colonies are shuffled among transects in both habitats combined to remove both within-habitat spatial separation and habitat specialization. For details, see Methods: Construction of random models.

which removed just the effects of within-habitat spatial separation on community similarity (see Construction of random models). If hypothesis 3 is correct, then community similarity in this randomization should increase relative to observed community similarity as scale increases. We also evaluated habitat specialization by comparing community similarity generated by the first randomization model with that generated by the second. If the former is higher than the latter, significant habitat specialization occurs at that scale.

\section{Construction of random models}

We used standard randomization methods (Gotelli and Graves 1996, Gotelli and Colwell 2001, Gering and Crist 2002, Crist et al. 2004, Freestone and Inouye 2006) to construct the random models. We used individualbased randomizations and random draws of transects within and among habitats to partition the relative effects of within-habitat spatial separation and habitat specialization on observed community similarity.
To remove the effects of within-habitat spatial separation, we used individual-based randomizations within each of the three habitats. Individual colonies were randomly shuffled 100 times among the 10 transects at each site (Fig. 2), the 40 transects on each island, and the 120 transects in each island group, keeping the number of colonies in each transect constant. After each shuffle, one transect was randomly drawn from the pool of 10 at each site for the transectsite scale (Fig. 2). Ten and 40 were drawn from pools of 40 and 120 on each island and in each island group for the site-island and island-island group scales, respectively. New $\alpha$ diversities were then calculated as the mean of the 100 random draws from each transect pool (Fig. 2). We then determined community similarity by averaging $\alpha$ diversity across the three habitats and regressing these on $\gamma$ diversity, just as was done for observed community similarity (Fig. 3). Community similarity with just the effects of within-habitat spatial separation removed was designated $p_{\mathrm{wh}}$ (Fig. 3). 


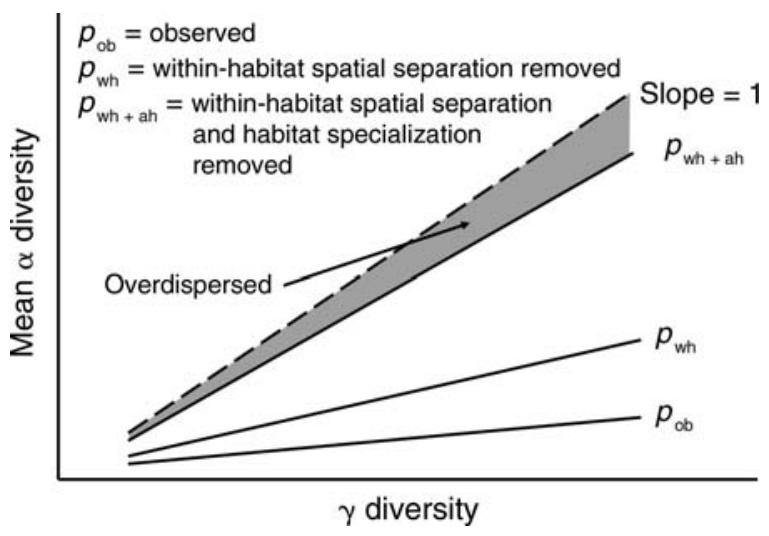

FIG. 3. Hypothetical regressions of mean $\alpha$ vs. $\gamma$ diversity for observed samples $\left(p_{\mathrm{ob}}\right)$, samples randomized within habitats $\left(p_{\mathrm{wh}}\right)$, and samples randomized within and among habitats $\left(p_{\text {wh+ah }}\right)$. Slopes show mean community similarity over all values of $\gamma$ diversity. For $p_{\mathrm{ob}}$ and $p_{\mathrm{wh}}, \alpha$ diversities were averaged over all three habitats before running the regressions to avoid pseudoreplication. The differences of $p_{\mathrm{wh}}-p_{\mathrm{ob}}$ and $p_{\mathrm{wh}}$ $-p_{\mathrm{wh}+\mathrm{ah}}$ indicate the effects of within-habitat spatial separation and habitat specialization on $p_{\text {ob }}$. The slope of the regression labeled $p_{\text {wh+ah }}$ represents community similarities for totally randomized samples. Slopes higher than this would therefore represent community similarities for species distributions that are more regular than random. Such regressions would fall in the region of the graph labeled overdispersed.

To remove the effects of both within-habitat spatial separation and habitat specialization, transects were pooled among all three habitats. Thirty, 120, and 360 transects were pooled at the site, island, and island group scales, respectively. To keep sample sizes consistent with the within-habitat randomizations, 10, 40, and 120 transects were randomly drawn from the pool at each scale, respectively. Individual colonies were then

TABLE 1. The effects of within-habitat spatial separation and habitat specialization on community similarity at the transect-site scale.

\begin{tabular}{lcccc}
\hline \hline \multicolumn{1}{c}{ Model } & $\begin{array}{c}\Delta \text { Community } \\
\text { similarity }\end{array}$ & SE & $t$ & $P$ \\
\hline$p_{\text {ob }}-0 \ddagger$ & 0.085 & 0.011 & 7.444 & $<0.00001$ \\
$p_{\text {wh }}-p_{\text {ob } \S}$ & 0.025 & 0.016 & 1.561 & 0.1202 \\
$p_{\text {wh }}-p_{\text {wh }+ \text { ah }} \|$ & 0.015 & 0.018 & 0.839 & 0.4032 \\
$p_{\text {wh }+ \text { ah }}-p_{\text {ob }}$ & 0.040 & 0.016 & 2.521 & 0.0126 \\
\hline
\end{tabular}

Notes: Differences were tested with one-sample, two-tailed $t$ statistics $\left(p_{\mathrm{ob}}-0\right)$ and with two-sample, two-tailed $t$ statistics (all other models). Model terms are as follows: $p$, slope of the plot of $\alpha$ vs. $\gamma$ diversity (index of mean community similarity across regions and a basis for inferring how $\beta$ diversity varies with spatial scale); $p_{\mathrm{ob}}$, observed community similarity; $p_{\mathrm{wh}}$, community similarity with just the effects of within-habitat spatial separation removed; $p_{\mathrm{wh}+\mathrm{ah}}$, community similarity with the effects of both within-habitat spatial separation and habitat specialization removed.

$\uparrow$ Differences are between regression coefficients.

\$ Observed community similarities.

$\S$ Independent effects of within-habitat spatial separation.

|| Independent effects of habitat specialization.

- Effects of within-habitat spatial separation and habitat specialization combined.
TABLE 2. The effects of within-habitat spatial separation and habitat specialization on community similarity at the siteisland scale.

\begin{tabular}{lcccc}
\hline \hline \multicolumn{1}{c}{ Model } & $\begin{array}{c}\Delta \text { Community } \\
\text { similarity } \dagger\end{array}$ & SE & $t$ & $P$ \\
\hline$p_{\text {ob }}-0 \ddagger$ & 0.261 & 0.018 & 14.776 & $<0.00001$ \\
$p_{\text {wh }}-p_{\text {ob }}$ & 0.063 & 0.025 & 2.520 & 0.0159 \\
$p_{\text {wh }}-p_{\text {wh }+ \text { ah }} \|$ & 0.060 & 0.026 & 2.329 & 0.0279 \\
$p_{\text {wh }+ \text { ah }}-p_{\text {ob }} \|$ & 0.123 & 0.025 & 4.908 & $<0.00001$ \\
\hline
\end{tabular}

Note: See Table 1 for explanation of model terms.

$\uparrow$ Differences are between regression coefficients.

\$ Observed community similarities.

$\S$ Independent effects of within-habitat spatial separation.

|| Independent effects of habitat specialization.

- Effects of within-habitat spatial separation and habitat specialization combined.

shuffled among transects across habitats in these randomized samples. After each shuffle, 1, 10, and 40 transects were randomly drawn from the site, island, and island group pools, respectively. New $\alpha$ diversities and community similarities were determined as before. Because habitats were combined in this randomization 60,15 , and 5 values for $\alpha$ diversity were generated at the transect-site, site-island, and island-island group scales, respectively. To standardize the number of values used to calculate $p_{\mathrm{ob}}$ and $p_{\mathrm{wh}}$ (where habitats were not combined) with these, $\alpha$ diversities were averaged across all three habitats for $p_{\mathrm{ob}}$ and $p_{\mathrm{wh}}$ as just indicated. We designated community similarity with the effects of both within-habitat spatial separation and habitat specialization removed, $p_{\mathrm{wh}+\mathrm{ah}}$ (Fig. 3).

To test hypothesis 1 , observed community similarities $\left(p_{\mathrm{ob}}-0\right)$ were tested for significance at all three scales using one-sample, two-tailed $t$ tests, and differences among scales were tested with two-sample, two-tailed $t$ tests. To test hypothesis 2 , the effects of underdispersion on community similarity, $p_{\mathrm{wh}+\mathrm{ah}}-p_{\mathrm{ob}}$, were tested for significance at each scale using two-sample, two-tailed $t$ tests. To test hypothesis 3, the independent effects of within-habitat spatial separation, $p_{\mathrm{wh}}-p_{\mathrm{ob}}$, and habitat specialization, $p_{\mathrm{wh}}-p_{\mathrm{wh}+\mathrm{ah}}$, on community similarity were tested for significance at each scale using this same statistic.

\section{Results}

Observed community similarity increased with spatial scale as predicted by hypothesis 1 . The slopes of the regressions $\left(p_{\mathrm{ob}}-0\right)$ became progressively steeper from the transect-site through the site-island to the islandisland group scales $(0.085,0.261,0.407$; Tables 1-3, Fig. 4). The site-island slope was significantly higher than the transect-site slope $(t=12.418, n=15, P<$ $0.00001)$ and island-island group slope was significantly higher than the site-island slope $(t=5.454, n=5, P<$ $0.0001)$. Values of $\bar{\beta}_{1}(0.915,0.739$, and 0.593$)$ correspondingly decreased with scale.

As predicted by hypothesis 2, the effects of underdispersion on community similarity were significant at 
TABLE 3. The effects of within-habitat spatial separation and habitat specialization on community similarity at the islandisland group scale.

\begin{tabular}{lcccc}
\hline \hline \multicolumn{1}{c}{ Model } & $\begin{array}{c}\Delta \text { Community } \\
\text { similarity } \dagger\end{array}$ & SE & $t$ & $P$ \\
\hline$p_{\text {ob }}-0 \ddagger$ & 0.407 & 0.023 & 17.890 & $<0.00001$ \\
$p_{\text {wh }}-p_{\text {ob } §}$ & 0.091 & 0.032 & 2.845 & 0.0192 \\
$p_{\text {wh }}-p_{\text {wh }+ \text { ah }} \|$ & 0.096 & 0.033 & 2.916 & 0.0268 \\
$p_{\text {wh }+ \text { ah }}-p_{\text {ob }} \uparrow$ & 0.188 & 0.032 & 5.835 & 0.0002
\end{tabular}

Note: See Table 1 for explanation of model terms.

$\uparrow$ Differences are between regression coefficients.

\$ Observed community similarities.

$\S$ Independent effects of within-habitat spatial separation.

|| Independent effects of habitat specialization.

- Effects of within-habitat spatial separation and habitat specialization combined.

all scales (Tables 1-3). The values for $p_{\mathrm{wh}+\mathrm{ah}}-p_{\mathrm{ob}}$ were $0.040,0.123$, and 0.188 , respectively. The slopes from these randomization models, $p_{\mathrm{wh}+\mathrm{ah}}$, are remarkably consistent, being 46-47\% higher than the observed values. These results indicate that some combination of within-habitat spatial separation and habitat specialization occurs at all three spatial scales. The independent contributions of these two components of underdispersion to community similarity will now be considered.

Although we expected that within-habitat spatial separation and habitat specialization should differ in the way they varied with scale, we found their effects on community similarity to be almost identical. Neither effect was statistically significant at the transect-site scale (Table 1), probably due to the conservative nature of our analysis. At the site-island and island-island group scales, both effects on community similarity were significant, with values of $p_{\mathrm{wh}}-p_{\mathrm{ob}}$ and $p_{\mathrm{wh}}-p_{\mathrm{wh}+\mathrm{ah}}$ that were $22-24 \%$ higher than observed at each scale (Tables 2-3). Thus, contrary to the prediction of hypothesis 3, the effect of within-habitat spatial separation on community similarity did not increase with scale. However, consistent with hypothesis 3 , the effects of habitat specialization were not different between the site-island and island-island group scales (Tables 2 and 3).

\section{Discussion}

Our finding that community similarity increased with spatial scale mirrors that of several other recent studies (Izsak and Price 2001, Lennon et al. 2001, Gering and Crist 2002, Freestone and Inouye 2006; but see Ellingsen 2001, Summerville et al. 2003). The increase indicates that a higher proportion of $\gamma$ diversity is subsumed by $\alpha$ diversity at each progressively larger scale and community similarity necessarily increases as well. This increase is caused by more rapid increases in $\alpha$ diversity accompanied by slower increases in $\gamma$ diversity as scale increases. This differential rate of increase of $\alpha$ probably occurs because the species-area relationship of Pacific corals on a double-logarithmic scale has a slope of less than 1 (Bellwood and Hughes 2001, Bellwood et al. 2005). Increases in $\alpha$ relative to $\gamma$ diversity with scale are expected in this circumstance (see Introduction).

Underdispersion significantly affected community similarity at all three scales. This result has consequences for the rate at which community similarity increases with spatial scale. If underdispersion either increases or decreases with increasing scale, then the rate of increase in community similarity will be diminished or enhanced relative to the case where species are randomly distributed in space. In this study, underdispersion was about the same at each scale, and thus observed community similarity increased at the same rate as in

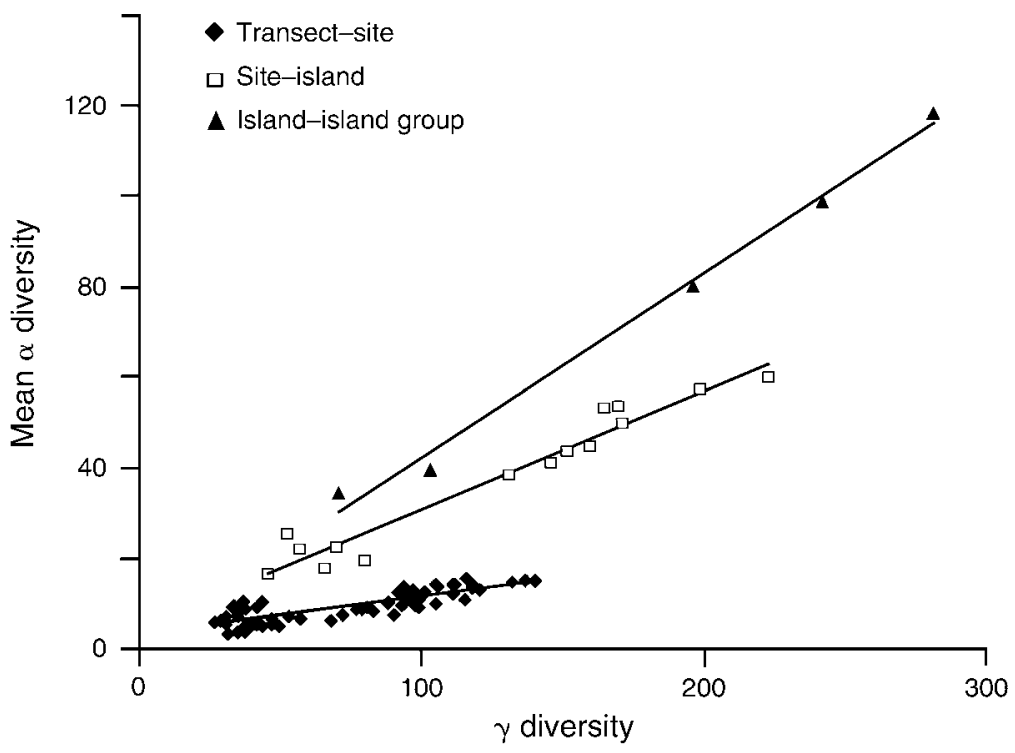

FIG. 4. Linear regressions of mean $\alpha$ diversity on $\gamma$ diversity at three different spatial scales. 


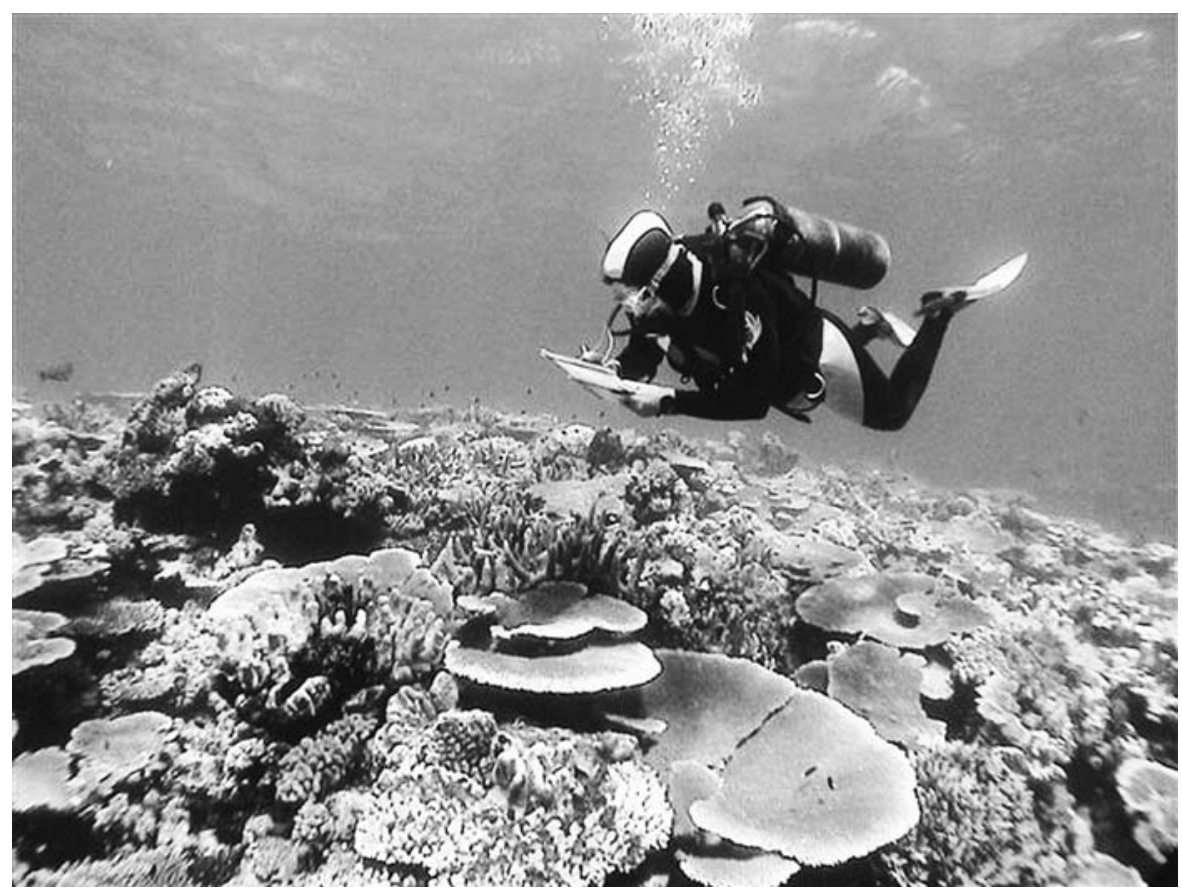

Plate 1. A diverse coral assemblage from northern Sulawesi, Indonesia, typical of reef crests and shallow reef slopes in the western Pacific biodiversity hotspot. Photo credit: T. Hughes.

the model which randomized species across sites and habitats.

When considered separately, habitat specialization and within-habitat spatial separation also had similar effects on community similarity as scale increased. When the effects of habitat specialization were removed, the effect on community similarity differed little (23\%) at the site-island and island-island group scales (Tables 2 and 3). The null expectation is that habitat specialization should not change appreciably with spatial scale because habitat differences are the same regardless of scale. Deviations from this expectation might occur if the numbers of species or the numbers of colonies in different habitat types follow different trajectories within or between scales. The fact that our results support the null expectation suggests that these differences do not occur. Of the various mechanisms that can cause habitat specialization, habitat selection during propagule settlement (Mundy and Babcock 2000, Carlon 2002, Baird et al. 2003), biotic factors such as fish predation (Wellington 1982), or adaptation to different abiotic environments (e.g., Done 1983, Smith and Hughes 1999) might all play a role. Interspecific interactions might, in theory, exclude species from some habitats, but there is little evidence supporting this mechanism in coral assemblages (McCook et al. 2001, Connell et al. 2004). Habitat restriction may also result from chance habitat occupancy of very rare species represented by one or a few colonies. However, our measure of habitat specialization is relatively insensitive to rarity-caused habitat restriction because the random- izations simply reshuffle rare species to different habitats without appreciably changing the range of habitats they occupy. The habitat specialization detected by the randomization models is thus likely to have biological rather than stochastic explanations.

The effects of within-habitat spatial separation on community similarity were also similar as scale increased (23-24\% at the site-island and island-island group scales). Within-habitat spatial separation can result from dispersal-extinction dynamics in fragmented landscapes, aggregated settlement of propagules, facilitative or preemptive interactions among species, and other clustering processes. Aggregated settlement and species interactions are expected to operate only at smaller scales. The former almost certainly plays a role at the transect-site scale (e.g., Lewis 1974), but the latter is unlikely to be important in coral assemblages (Cornell and Karlson 2000). Spatial separation due to dispersalextinction dynamics is expected to operate at larger scales and has been shown to be important in other insular systems (Harrison 1997, Freestone and Inouye 2006). It has also been shown to increase with spatial scale in at least one study (Freestone and Inouye 2006). Islands within island groups are, by definition, farther apart from one another than reef sites on islands, and their increased isolation should present a greater challenge to the dispersal ability of coral propagules. If this is the case, then coral populations are less likely to be rescued from extinction as scale increases, and the effect of spatial separation on community similarity should increase with spatial scale in the coral system as 
well. The fact that the effect of spatial separation is roughly the same across scales suggests that although dispersal limitation relative to extinction probably occurs in this system, the isolation that occurs at the island-island group scale relative to the site-island scale does not present a serious additional obstacle to colonization by a coral species. It is not particularly surprising that dispersal limitation does not increase from the island to the island-groups scale because most corals have pelagic larvae. They can begin to settle 1-4 days after broadcast spawning but may remain competent for much longer. The implication is that, at relevant time scales, coral species can disperse as easily over the distances that separate islands within island groups as those that separate sites on islands.

Within-habitat spatial separation almost certainly reduces community similarity among island groups as well. The number of endemic species of corals is greatest in the Indo-Australian hotspot (Hughes et al. 2002, Roberts et al. 2002). This pattern could be explained in part by a combination of reduced dispersal and higher extinction in the central Pacific. The $10000-\mathrm{km}$ extent of the gradient we sampled and the direction of prevailing currents are more than sufficient to limit the dispersal of pelagic propagules to more isolated parts of the Pacific (Connolly et al. 2003, Dornelas et al. 2006). In addition, reef area is greatest within the central Indo-Pacific hotspot (Bellwood and Hughes 2003, Bellwood et al. 2005), effectively increasing mean coral population sizes and likely reducing their susceptibility to extinction. Conversely, many coral species may not be present in the central Pacific because they have not gotten there or because the rate of extinction exceeds the rate of rescue by dispersal. Thus, community similarities among the eastern and western island groups along our gradient are likely to be lower than if species were randomly distributed among island groups.

Neither habitat specialization nor within-habitat spatial separation significantly affected community similarity at the transect-site scale (although in combination they were significant; Table 1). Part of the explanation is the conservative nature of the analysis. Since we averaged $\alpha$ diversities across habitats to avoid pseudoreplication and to keep all randomization analyses consistent, our sample size was 60 at this scale. A more sensitive analysis that did not average across habitats, and thus had a sample size of 180 , found significant within-habitat spatial separation at this scale (Karlson et al. 2007).

Ours is the first study to simultaneously measure the effects of underdispersion on community similarity across scales and among regions. It is also the first examination of these patterns in a marine system. We show that underdispersion significantly reduces community similarity and thus increases $\beta$ diversity at all scales examined, which means that, ceteris paribus, preservation of coral biodiversity will require areas larger than predicted by models that assume random dispersion.
Moreover, our suggested explanations for reduced community similarity at different scales now need rigorous testing in order to understand the underlying causes for the spatial structure that characterizes reef assemblages.

\section{ACKNOWLEDGMENTS}

We acknowledge Jackie Wolstenholme, Mary Jo Boyle, Liz Dinsdale, Alison Green, Andrew Baird, Sean Connolly, Marie Kospartov, Phil Osmond, and Kim Osmond for their assistance in the field, and Andrew Baird and Susan Harrison for critically reading the manuscript. Funding was provided by grants from the Australian Research Council, the National Science Foundation (International Programs and the Ocean Sciences Division), and the National Geographic Society.

\section{Literature Cited}

Baird, A. H., R. C. Babcock, and C. P. Mundy. 2003. Habitat selection by larvae influences the depth distribution of six common coral species. Marine Ecology Progress Series 252: 289-293.

Bellwood, D. R., and T. P. Hughes. 2001. Regional-scale assembly rules and biodiversity of coral reefs. Science 292: $1532-1534$.

Bellwood, D. R., T. P. Hughes, S. R. Connolly, and J. Tanner. 2005. Environmental and geometric constraints on IndoPacific coral reef biodiversity. Ecology Letters 8:643-651.

Carlon, D. B. 2002. Production and supply of larvae as determinants of zonation in a brooding tropical coral. Journal of Experimental Marine Biology and Ecology 268: $33-46$.

Clarke, A., and S. Lidgard. 2000. Spatial patterns of diversity in the sea: bryozoan species richness in the North Atlantic. Journal of Animal Ecology 69:799-814.

Cody, M. L. 1993. Bird diversity components within and between habitats in Australia. Pages 147-158 in R. E. Ricklefs and D. Schluter, editors. Species diversity in ecological communities: historical and geographical perspectives. University of Chicago Press, Chicago, Illinois, USA.

Connell, J. H., T. P. Hughes, C. C. Wallace, J. E. Tanner, K. E. Harms, and A. M. Kerr. 2004. A long-term study of competition and diversity of corals. Ecological Monographs 74:179-210.

Connolly, S. R., D. R. Bellwood, and T. P. Hughes. 2003. IndoPacific biodiversity of coral reefs: deviations from a middomain model. Ecology 84:2178-2190.

Cornell, H. V., and R. H. Karlson. 2000. Coral species richness: ecological vs. biogeographic influences. Coral Reefs 19:37-49.

Cornell, H. V., and J. H. Lawton. 1992. Species interactions, local and regional processes, and limits to the richness of ecological communities: a theoretical perspective. Journal of Animal Ecology 61:1-12.

Crist, T. O., J. A. Veech, J. C. Gering, and K. S. Summerville. 2004. Partitioning species diversity across landscapes and regions: a hierarchical analysis of $\alpha, \beta$, and $\gamma$ diversity. American Naturalist 162:734-743.

Done, T. J. 1983. Coral zonation: its nature and significance. Pages 95-147 in B. J. Barnes, editor. Perspectives on coral reefs. Brian Clouston, Manuka, Australia.

Dornelas, M., S. R. Connolly, and T. P. Hughes. 2006. Coral reef diversity refutes the neutral theory of biodiversity. Nature 440:80-82.

Edinger, E. N., J. Kolasa, and M. J. Risk. 2000. Biogeographic variation in coral species diversity on coral reefs in three regions of Indonesia. Diversity and Distributions 6:113-127.

Ellingsen, K. E. 2001. Biodiversity of a continental shelf softsediment macrobenthos community. Marine Ecology Progress Series 218:1-15. 
Ellingsen, K. E., and J. S. Gray. 2002. Spatial patterns of benthic diversity: is there a latitudinal gradient along the Norwegian continental shelf? Journal of Animal Ecology 71: 373-389.

Freestone, A. L., and B. D. Inouye. 2006. Dispersal limitation and environmental heterogeneity shape scale-dependent diversity patterns in plant communities. Ecology 87:24252432.

Gaston, K. J., and P. H. Williams. 1996. Spatial patterns in taxonomic diversity. Pages 202-229 in K. J. Gaston, editor. Biodiversity: a biology of numbers and differences. Blackwell Science, Oxford, UK.

Gering, J. C., and T. O. Crist. 2002. The alpha-beta-regional relationship: providing new insights into local-regional patterns of species richness and scale dependence of diversity components. Ecology Letters 5:433-444.

Gering, J. C., T. O. Crist, and J. A. Veech. 2003. Additive partitioning of species diversity across multiple spatial scales: implications for regional conservation of biodiversity. Conservation Biology 17:488-499.

Gotelli, N. J., and R. K. Colwell. 2001. Quantifying biodiversity: procedures and pitfalls in the measurement and comparison of species richness. Ecology Letters 4:379-391.

Gotelli, N. J., and G. R. Graves. 1996. Null models in ecology. Smithsonian Institution, Washington, D.C., USA.

Green, J. L., and A. Ostling. 2003. Endemics - area relationships: the influence of species dominants and spatial aggregation. Ecology 84:3090-3097.

Harrison, S. 1997. How natural habitat patchiness affects the distribution of diversity in Californian serpentine chaparral. Ecology 78:1898-1906.

Harrison, S. 1999. Local and regional diversity in a patchy landscape: native, alien, and endemic herbs on serpentine soils. Ecology 80:70-80.

Harrison, S., S. J. Ross, and J. H. Lawton. 1992. Beta diversity on geographic gradients in Britain. Journal of Animal Ecology 61:141-148.

He, F., and P. Legendre. 2002. Species diversity patterns derived from species-area models. Ecology 83:1185-1198.

Hillebrand, H. 2004. On the generality of the latitudinal diversity gradient. American Naturalist 163:192-211.

Hughes, T. P., D. R. Bellwood, and S. R. Connolly. 2002. Biodiversity hotspots, centers of endemicity, and the conservation of coral reefs. Ecology Letters 5:775-784.

Izsak, C., and J. R. G. Price. 2001. Measuring $\beta$-diversity using a taxonomic similarity index and its relation to spatial scale. Marine Ecology Progress Series 215:69-77.

Karlson, R. H., H. V. Cornell, and T. P. Hughes. 2004. Coral communities are regionally enriched along an oceanic biodiversity gradient. Nature 429:867-870.

Karlson, R. H., H. V. Cornell, and T. P. Hughes. 2007. Aggregation influences coral species richness at multiple spatial scales. Ecology 88:170-177.

Koleff, P., J. J. Lennon, and K. J. Gaston. 2003. Are there latitudinal gradients in species turnover? Global Ecology and Biogeography 12:483-498.

Lande, R. 1996. Statistics and partitioning of species diversity and similarity among multiple communities. Oikos 76:5-13.

Lennon, J. J., P. Koleff, J. J. D. Greenwood, and K. J. Gaston. 2001. The geographical structure of British bird distributions: diversity, spatial turnover, and scale. Journal of Animal Ecology 70:966-979.

Lewis, J. B. 1974. The settlement behaviour of planula larvae of the hermatypic coral Favia fragum (Esper). Journal of Experimental Marine Biology and Ecology 15:165-172.

Loreau, M. 2000. Are communities saturated? On the relationship between alpha, beta, and gamma diversity. Ecology Letters 3:73-76.

McCook, L. J., J. Jompa, and G. Diaz-Pulido. 2001. Competition between corals and algae on coral reefs: a review of evidence and mechanisms. Coral Reefs 19:400-417.

Mundy, C., and R. Babcock. 2000. Are vertical distribution patterns of scleractinian corals maintained by pre- or postsettlement processes? A case study of three contrasting species. Marine Ecology Progress Series 198:109-119.

Okuda, T., T. Noda, T. Yamamoto, N. Ito, and M. Nakaoda. 2004. Latitudinal gradient of species diversity: multi-scale variability in rocky intertidal sessile assemblages along the Northwestern Pacific coast. Population Ecology 46:159-170.

Qian, H., R. E. Ricklefs, and P. S. White. 2004. Beta diversity of angiosperms in temperate floras of eastern Asia and eastern North America. Ecology Letters 7:15-22.

Rivadeneira, M. M., M. Fernández, and S. A. Navarrete. 2002. Latitudinal trends of species diversity in rocky intertidal herbivore assemblages: spatial scale and the relationship between local and regional species richness. Marine Ecology Progress Series 245:123-131.

Roberts, C. M., C. J. McClean, J. E. N. Veron, J. P. Hawkins, G. R. Allen, D. E. McAllister, C. G. Mittermeier, F. W. Schueler, M. Spalding, F. Wells, C. Vynne, and T. B. Werner. 2002. Marine biodiversity hotspots and conservation priorities for tropical reefs. Science 295:1280-1284.

Scheiner, S. M., S. B. Cox, M. Willig, G. G. Mittelbach, C. Osenberg, and M. Kaspari. 2000. Species richness, speciesarea curves, and Simpson's paradox. Evolutionary Ecology Research 2:791-802.

Smith, L. D., and T. P. Hughes. 1999. An experimental assessment of survival, re-attachment and fecundity of coral fragments. Journal of Experimental Marine Biology and Ecology 235:147-164.

Srivastava, D. S. 1998. Using local-regional richness plots to test for saturation: pitfalls and potentials. Journal of Animal Ecology 68:1-16.

Summerville, K. S., M. Boulware, J. A. Veech, and T. O. Crist. 2003. Spatial variation in species diversity and composition of forest Lepidoptera; patterns and implications for conservation. Conservation Biology 17:1045-1057.

Wagner, H. H., O. Wildi, and K. C. Ewald. 2000. Additive partitioning of plant species diversity in an agricultural mosaic landscape. Landscape Ecology 15:219-227.

Wellington, G. 1982. Depth zonation of corals in the Gulf of Panama: control and facilitation by resident reef fishes. Ecological Monographs 52:223-241.

Whittaker, R. H. 1960. Vegetation of the Siskiyou Mountains, Oregon and California. Ecological Monographs 30:279-338.

Witman, J. D., R. J. Etter, and F. Smith. 2004. The relationship between regional and local species diversity in marine benthic communities: a global perspective. Proceedings of the National Academy of Sciences (USA) 101:15664-15669. 